\title{
Comparative Analysis of Image Segmentation using Thresholding
}

\author{
Gurjeet Kaur, Rajiv Kumar
}

\begin{abstract}
Image segmentation is an important technology for image processing. It is a critical and essential component of an image analysis and/or pattern recognition system, and is one of the most difficult tasks in image processing. Image segmentation is the process by which we segment a given image into several parts so that we can further analyzed each of these parts present in the image. We can extract some information by analyzing them and this information is useful for high-level machine vision applications. In this paper, we are analyzing and evaluating the various types of thresholding techniques such as single-value thresholding, multiple- thresholding, adaptive thresholding, optimal thresholding and local thresholding. These different thresholding techniques are extensively used in image segmentation. We have taken into consideration the threshold value which is used to segment a given image. The experimental results show that each technique performs better depending on the different situations. The results are implementing and shown on various images using Image Processing Toolbox (IPT) in MATLAB.
\end{abstract}

Index Terms - Image segmentation, thresholding, adaptive thresholding, optimal thresholding, local thresholding.

\section{INTRODUCTION}

Image segmentation is considered as an important basic operation for meaningful analysis and interpretation of image acquired. Image segmentation is the process of dividing an image into different regions such that each region is homogeneous. A number of image segmentation techniques are available, but there is no one single technique that is suitable to all the applications. Researchers have extensively worked over this fundamental problem and proposed various methods for image segmentation. These methods can be broadly classified into seven groups: (1) Histogram thresholding, (2) Clustering (Fuzzy and Hard), (3) Region growing, Region splitting and merging, Discontinuity-based, (5) Physical model- based, (6) Fuzzy approaches, and (7) Neural network and GA (Genetic algorithm) based approaches. Thresholding is one of the fundamental approaches of image segmentation. In case of uniform image, the histogram has a deep and sharp valley between two peaks representing objects and background, respectively, so that the threshold can be chosen at the bottom of this valley. In this paper, we will analyze and evaluate the threshold techniques such as single-thresholding, multiple-thresholding, adaptive thresholding, optimal

\section{Gurjeet Kaur, Department of Computer, GHSS, Nilokheri, India.}

Rajiv Kumar, Faculty of Computing, Botho University, Gaborone, Botswana. thresholding and local thresholding. But, broadly, we can divide thresholding in two areas - global and local. In case of global thresholding, only one threshold has chosen based on the global information about the image. However, in case of local thresholding, the threshold has chosen based on the local properties of the pixels of an image.

The work in this paper is organized as: section II described the related work in the area of thresholding. In section III, we have analyzed and evaluated the various types of thresholding techniques. The different methods are tested on the various images. In section IV, we describe results and discussions of thresholding techniques along with the parameter values. Finally, section $\mathrm{V}$ concludes the remarks.

\section{RELATED WORK}

J. S. Weszka et al. [1] propose two methods for measuring the "goodness" of a threshold image, one based on a busyness criterion and the other based on a discrepancy or error criterion. These evaluation techniques are applied to a set of infrared images and are shown to be useful in facilitating threshold selection. In fact, both methods usually result in similar or identical thresholds which yield good segmentation of the image. N. Otsu [2] presents a nonparametric and unsupervised method of automatic threshold selection for picture segmentation. An optimal threshold is selected by the discriminant criterion, namely, so as to maximize the separability of the resultant classed in gray levels. I. E. Abdou et al. [3] developed an quantitative design and performance evaluation techniques for the enhancement/thresholding class of image edge detectors. The design techniques are based on statistical detection theory and deterministic pattern-recognition classification procedures. The performance evaluation methods developed include: a) deterministic measurement of the edge gradient amplitude; b) comparison of the probabilities of correct and false edge detection; and c) figure of merit computation. K. S. Fu et al. [4] summarized image segmentation techniques. These techniques can be categorized into three classes, (1) characteristic features thresholding or clustering, (2) edge detection, and (3) region extraction. R. M. Haralick [5] uses the facet model to accomplish step edge detection. He define an edge to occur in a pixel if and only if there is some point in the pixel's area having a negatively sloped zero crossing of the second directional derivative taken in the direction of a nonzero gradient at the pixel's center. N. R. Pal and S. K. Pal [6] reviews and summarizes image segmentation algorithms which are based on fuzzy set, neural networks, thresholding, edge detection, clustering relaxation and Markov Random Field (MRF). They also address the issue of quantitative evaluation of segmentation results. J. C. Yen et al. [7] propose 
a new criterion for multilevel thresholding. The criterion is based on the consideration of two factors. The first one is the discrepancy between the thresholded and original images and the second one is the number of bits required to represent the threshold image. Computational analyses indicate that the number of required mathematical operations in the implementation of proposed algorithm is much less than that of maximum entropy criterion. A.T. Abak et al. [8] present performance evaluation of thresholding algorithms in the context of document analysis and character recognition systems. Several thresholding algorithms are comparatively evaluated on the basis of the original bitmaps of characters. Different distance measures such as, Haussorff, Jaccard, and Yule are used to measure the similarity between thresholded bitmaps and original bitmaps of characters. F. H. Y. Chan et al. [9] propose a new adaptive thresholding method using variational theory. The method requires only one parameter to be selected and the adaptive threshold surface can be found automatically from the original image. C. Hampton et al. [10] summarized the various image segmentation techniques based on discontinuity-based, region-based, thresholding and histogram. M. Cheriet et al. [11] present a general recursive approach for image segmentation by extending Otsu's method. The new approach goes beyond segmenting only one bright object from an image to an approach that recursively segments the brightest object at each recursion, leaving the darkest object in the given digitized image. O. J. Tobias et al. [12] propose an approach to threshold the histogram according to the similarity between gray levels. Such a similarity is assessed through a fuzzy measures. In this way, they overcome the local minima that affect most of the conventional methods. The experimental results demonstrate the effectiveness of the proposed approach for both bimodal and multimodal histograms. L. Djerou et al. [13] propose an automatic multilevel thresholding approach, based on Binary Particle Swarm Optimization. The proposed approach automatically determines the "optimum" number of the thresholds, by optimizing a function which uses the gray level thresholds as parameters. P. D. Satya [14] proposed an improved particle swarm optimization (IPSO) based multilevel thresholding for the minimization of objective function. The chaotic sequences are included in the inertia weight factor of the PSO to improve the searching capability of the algorithm. The experimental results show that the proposed method can make optimal thresholding applicable in case of multilevel thresholding and the performances are better than those of some property-based multilevel thresholding methods. N. Natarajan et al. [15] propose an algorithm to remove the unwanted portions by choosing an appropriate threshold. A criterion is proposed to determine primary threshold based on Otsu threshold and average of the means of the two classes obtained using Otsu method. The results show that it outperforms Otsu thresholding algorithm and provides a valuable tool especially for the extraction of features from rock images, in addition to images that involve cracks. T. Zuva et al. [16] provides a survey of achievements, problems being encountered, and the open issues in the research area of image segmentation and usage of the techniques in different areas. They considered the techniques under the following three groups: Threshold-based, Edge-based and Region-based.

\section{THRESHOLDING TECHNIQUES}

\section{A. Single thresholding}

It is the simplest approach of image segmentation. Suppose, if we have images where every pixel has coded with eight bits, then we have intensity varying from 0 to 255 . We can decide threshold following some criteria. For example: we have threshold level 128 so that it decide that all the pixels having intensity value greater than 128 , it belong to some regions and those intensity values less than 128 , it belong to some other region. Let an image be $f(x, y)$. Suppose that this image consists the dark object against the bright background or vice-versa. Therefore, intensity concentrate mainly on two regions, one towards the darker side (or lower intensity) and other towards the brighter side (or higher intensity). The histogram with two peaks and valley at the bottom as shown in the figure 1 .

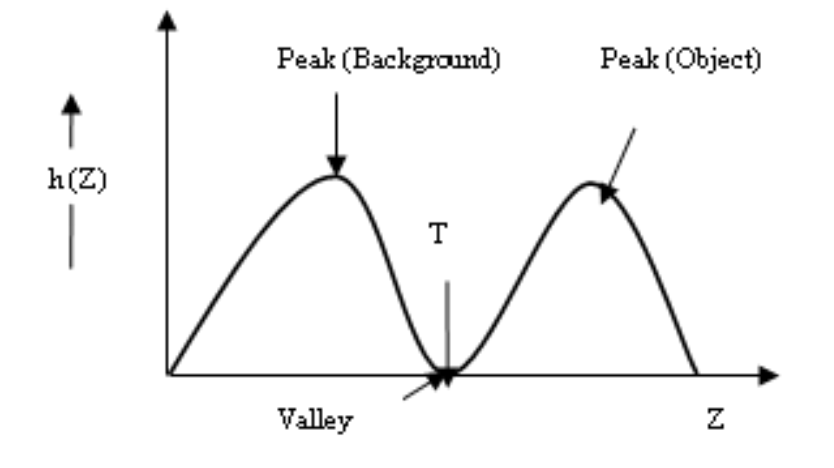

Fig. 1 Histogram with two peaks and one valley

It is also called Bi-model histogram.

$$
f(x)=\left\{\begin{array}{cr}
>T_{x} & \text { pixels belong to object } \\
\ll T_{x} & \text { pixels belong to bockground }
\end{array}\right.
$$

Where $\mathrm{T}$ is called the Threshold value. As shown in figure 2 , the image has a number of objects (coins) against the dark background. We have chosen the threshold value which is equal to 80 , to segment the given image.
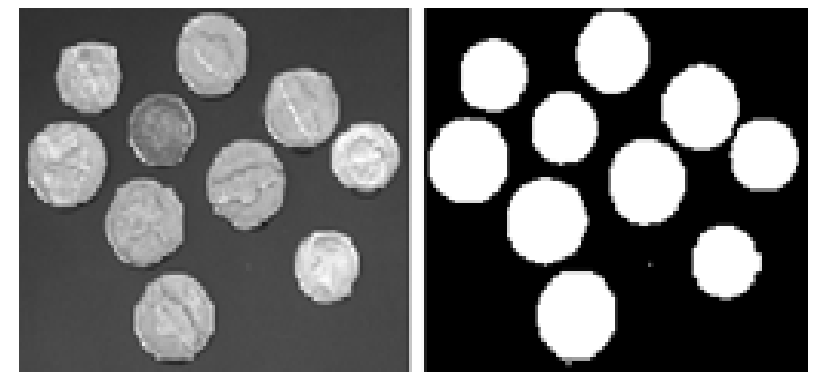

Fig. 2 Thresholding image of coins against the dark background

Another image which have the number of objects against the bright background. In that case, the threshold value is 99 . 


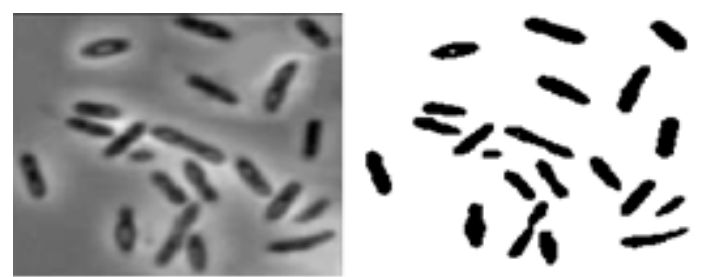

Fig. 3 Thresholding object against the bright background

\section{B. Multiple Thresholding}

In case of multi-model histogram, we have two objects and one background with two threshold values T1 and T2 as shown in figure 4.

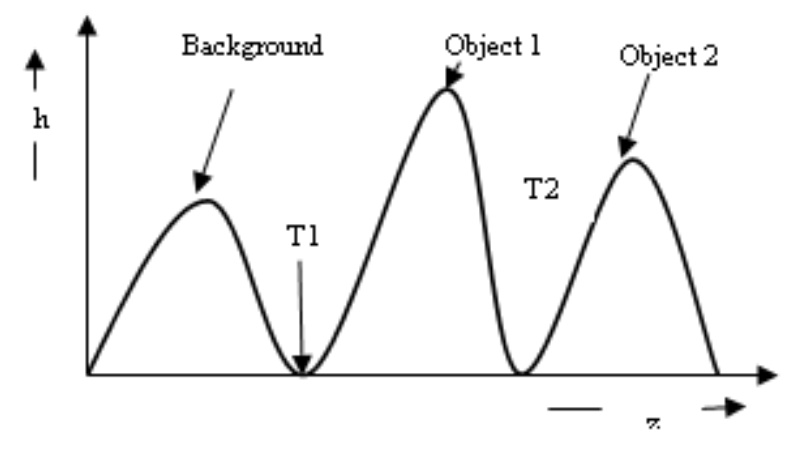

Fig. 4 Multiple Thresholding

$\left\{\begin{array}{c}f(x, y)>T 2 \Rightarrow(x, y) \text { belongs to object } 2 \\ T 1<f(x, y) \leq T 2 \Rightarrow(x, y) \text { belongs to object } 1 \\ f(x, y) \ll T 1 \Rightarrow(x, y) \text { belongs to background }\end{array}\right.$

Where T1 and T2 are the two threshold values. Figure 5 considers the image which have dark image within the object and this object has dark background. In that case, we have obtained two threshold values (115 and 125) to segment the image.
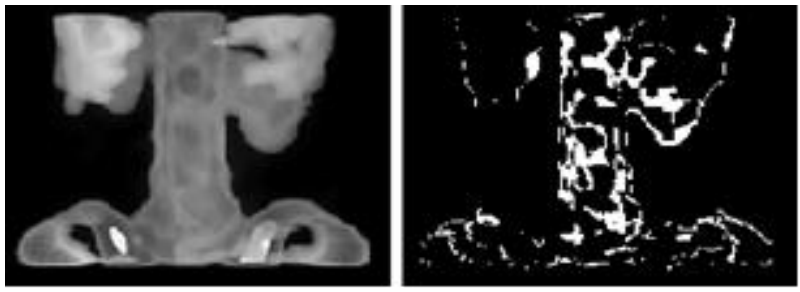

Fig. 5 Multiple thresholding

The important point is that we have to choose proper threshold value. This threshold value can be considered as an operation that involves testing against function $\mathrm{T}$ where this function $\mathrm{T}$ is of the form:

\section{$T=T\left[\begin{array}{lll}x_{v} y_{x} & p\left(x_{v} y\right) & f\left(x_{v} y\right)\end{array}\right]$}

Where $(\mathrm{x}, \mathrm{y})$ is the location of point in an image, $\mathrm{f}(\mathrm{x}, \mathrm{y})$ is the pixel intensity at location $(x, y)$ and $p(x, y)$ is local property in a neighborhood centered at $(x, y)$.

Therefore, $T$ is a combination of $(x, y), f(x, y)$ and $p(x, y)$ and depending upon these combination, $\mathrm{T}$ can be global, local or adaptive threshold. We have
$\mathrm{T}[\mathrm{f}(\mathrm{xy})] \Rightarrow \mathrm{T}$ is global threshold,

$\mathrm{T}[\mathrm{f}(\mathrm{x}, \mathrm{y}), \mathrm{p}(\mathrm{x}, \mathrm{y})] \Rightarrow \mathrm{T}$ is local threshold and

$\mathrm{T}[\mathrm{x}, \mathrm{y}, \mathrm{f}(\mathrm{x}, \mathrm{y}), \mathrm{p}(\mathrm{x}, \mathrm{y})] \Rightarrow \mathrm{T}$ is adaptive threshold.

Here, the value of T has chosen manually, but what happen when $\mathrm{T}$ has chosen automatically.

\section{Automatic Thresholding}

There are number of steps to be followed:

Step 1: Chose initial value of $T$.

Step 2: Group the intensities into G1 and G2.

Step 3: Calculate the mean $\mu 1$ and $\mu 2$ of $\mathrm{G} 1$ and G2 respectively.

Step 4: Calculate the threshold T such that

$$
T=\frac{\mu 1+\mu 2}{2}
$$

Step 5: Go to step 2, until $\left|T_{i}-T_{i+1}\right| \ll T^{v}$

Step 6: stop

It may be observed from the experiment that whatever the threshold we chose automatically, the automotive threshold will be similar to manual threshold. For the entire image, we choose one particular threshold and using this threshold, we go for segmenting the image, and this kind of threshold operation is called global threshold operation. It gives very good result if the intensity is uniform. But, if the intensity is not uniform, then the global threshold operation does not give the good results.

\section{Adaptive Thresholding}

To solve above problem, we sub-divide the image into number of smaller sub-images. Assume that in each of these sub-images, the intensity will be more or less uniform. We can easily find out the threshold value for each of these sub-images. Using this threshold value, we can threshold the sub-images and take union of all these threshold value, it gives the final threshold value. In this case, the threshold selection is position dependent. That why, it is called adaptive thresholding. As shown in figure 6, the image has variation in gray-level from dark to background. If we take the global thresholding, the result is very poor as shown in figure 6 (b). But, if we sub-divide the image into four regions and take the separate threshold of each region and finally, take the union of all these thresholds, we will get the results as shown in figure 6 (c). The result is much better as compare to figure 6 (b).
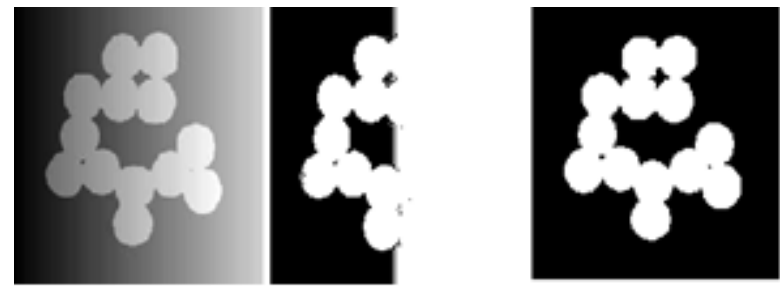

Fig. 6 Adaptive Thresholding 
Why adaptive thresholding is not successful for all regions in sub-images?

Let us take an example as shown in figure 7.

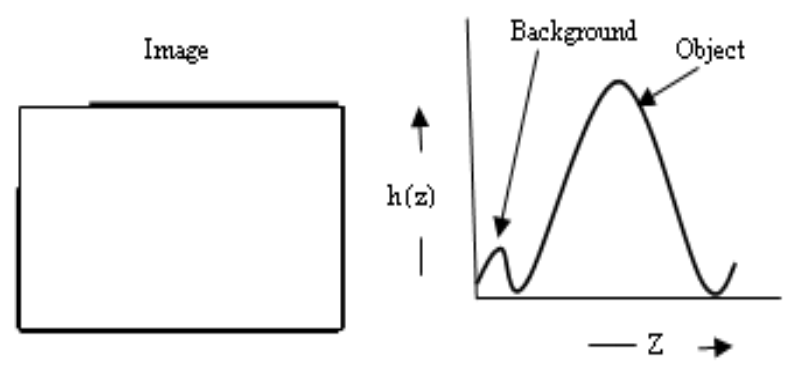

Fig. 7 Image and its Histogram

Suppose the small portion belong to background and large portion of the image is belonging to the object. If we plot the histogram, because the number of pixels in the background is very small so the contribution of these pixels of this histogram is almost negligible. Therefore, instead of becoming bi-model histogram, it is dominated by single peak. That why, this thresholding operation does not give good result for that region.

\section{How to solve this problem?}

We subdivide the image into sub-regions and for each of these sub-division, we try to find out the separate threshold for each region.

Problem: These thresholds do not tell about the accuracy of the threshold. Therefore, we use optimal thresholding which use the statistical property of the image, where mean error of the thresholding operation will be minimum.

\section{E. Optimal Thresholding}

Assume that the image consist two principle gray levels. One corresponds to object and other corresponds to background. We use a random variable $\mathrm{z}$ and normalized histogram of this variable $\mathrm{z}$ can be viewed as probability density function (PDF) which is represented by $\mathrm{p}(\mathrm{z})$.

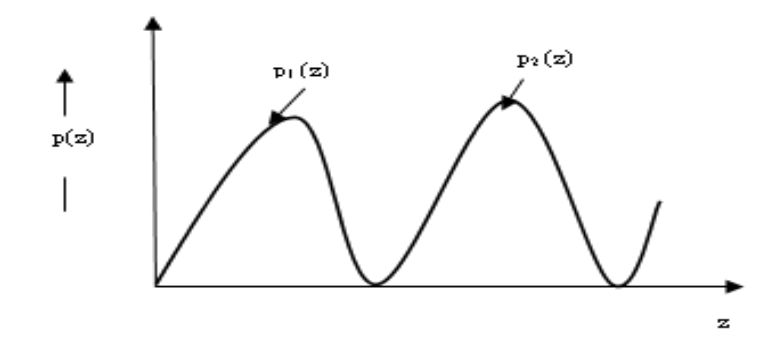

Fig. 8 Histogram with correspond to two gray-levels

This histogram is the combination of two PDFs. The p1 (z) and p2 $(\mathrm{z})$ is the probability density function belong to background and object respectively.

$\mathrm{p}(\mathrm{z})=\mathrm{P} 1 \mathrm{p} 1(\mathrm{z})+\mathrm{P} 2 \mathrm{p} 2(\mathrm{z})$

Where $\mathrm{P} 1$ and $\mathrm{P} 2$ are the probability belong to background and object respectively i.e. $\mathrm{P} 1+\mathrm{P} 2=1$. Here, we want to determine the threshold $\mathrm{T}$ which will minimize the average segmentation error. The overall probability is modeled as two different probabilities.

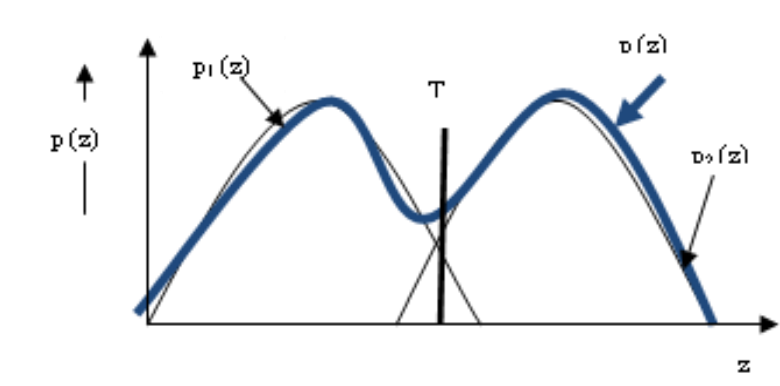

Fig. 9 Probability Density Function (PDF)

If $\mathrm{f}(\mathrm{x}, \mathrm{y})>\mathrm{T}$, then $(\mathrm{x}, \mathrm{y})$ object. Here, $\mathrm{T}$ is the threshold. Suppose $\mathrm{f}(\mathrm{x}, \mathrm{y})$ also having finite probability that it may be belong to background. So, by choosing this threshold, we also incorporating some error. The error is that the probability of considering the background point as object point. Probability of object pixels may be classified as

$$
E_{1}(T)=\int_{-\infty}^{+\infty} p_{2}(z) d
$$

Similarly, if the background pixel is classified as the object pixel, then the corresponding error will be given by

$$
E_{2}(T)=\int_{-\infty}^{+\infty} p_{1}(z) d z
$$

From these two equations, the overall error probability will be

$$
\mathrm{E}(\mathrm{T})=\mathrm{P} 2 \mathrm{E} 1(\mathrm{~T})+\mathrm{P} 1 \mathrm{E} 2(\mathrm{~T})
$$

Where P1 and P2 are the probability of pixels belong to background and object respectively.

To minimize the error, we have

$$
\frac{\partial E(T)}{\partial T}=0
$$

It will give minimum error. Therefore,

$$
P_{1} p_{1}(T)=P_{2} p_{2}(T)
$$

If we assume Gaussian PDF, then

$$
p(z)=\frac{P_{1}}{\sqrt{2} \pi \sigma_{1}} e^{-\frac{\left(z-\mu_{1}\right)^{2}}{2 \sigma^{2}}}+\frac{P_{2}}{\sqrt{2} \pi \sigma_{2}} e^{-\frac{\left(z-\mu_{2}\right)^{2}}{2 \sigma^{2}}}
$$

Where $\mu 1$ and $\mu 2$ are average intensity value of background and object regions respectively. $\sigma 1$ and $\sigma 2$ are the standard deviation of intensity value in background and object respectively.

The solution of threshold $(\mathrm{T})$ is given by

$\mathrm{AT} 2+\mathrm{BT}+\mathrm{C}=0$

Where $\mathrm{A}=\sigma_{1}^{2}+\sigma_{2}^{2}$

$\mathrm{B}=2\left(\mu_{1} \sigma_{2}^{2}+\mu_{2} \sigma_{1}^{2}\right)$

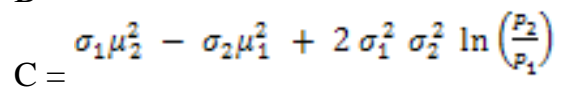

Assume that $\sigma_{1}^{2}=\sigma_{2}^{2}=\sigma^{2}$, then

$T=\frac{\mu_{1}+\mu_{2}}{2}+\frac{\sigma^{2}}{\mu_{1}-\mu_{2}} \ln \left(\frac{P_{2}}{P_{1}}\right)$

It gives the optimal results. If $\mathrm{P} 1=\mathrm{P} 2$, then 
$T=\frac{\mu_{1}+\mu_{2}}{2}$

Figure 10 shown an image which is using the optimal thresholding.

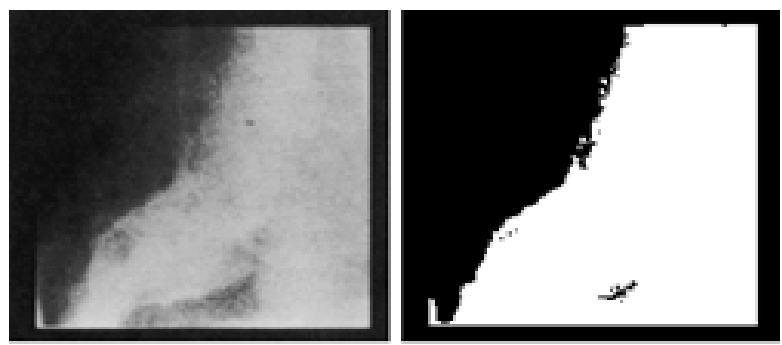

Fig. 10 Example of optimal thresholding

We consider another example as shown in figure 11 .

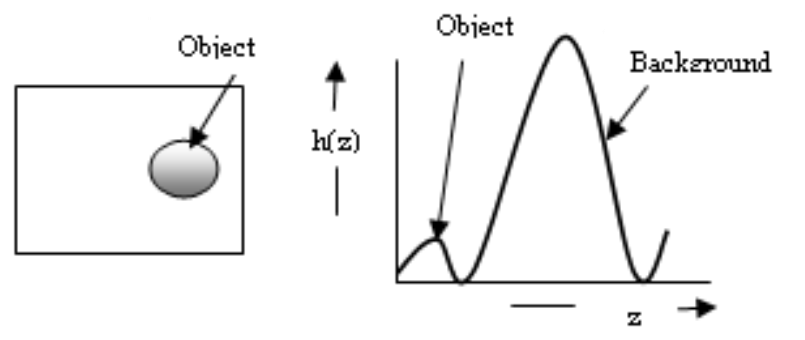

Fig. 11 Image and its Histogram

Suppose if we have very small number of pixels belong to object and large number of pixels belong to background. So, we will get histogram as shown in figure 11 . This problem can be solved if instead of considering all the pixels in the image to produce the histogram if somehow we identify the pixels which are either on the boundary or near the boundary between the object and the background. Since we considering only small pixels near the boundary, then few things occur which are:

- Histogram will be symmetric i.e. area of the pixels within the object region and the area of the pixels within the background region are almost same.

- The probability of pixels belonging to the object and background within this narrow region are almost equal.

Therefore, the histogram will be symmetric so that the thresholding operation is very simple.

Problem: If we use this simple approach, then we should know what the object boundary is or what is the boundary between the object region and the background region?

Solution: This is not easily obtained because the basic purpose of segmentation is that we are tried to do find out the boundary between the object and background. So, this could not be used because boundary itself is not known.

\section{F. Local Thresholding}

To obtain the solution of above problem, we use image Gradient and image Laplacian. We know that an estimate of edge points can be obtained from the Gradient and Laplacian operator. Laplacian operator is affected to a large extent by the presence of noise so that the output of the Laplacian operator is not directly used for edge detection but it is used to provide secondary information. We determine the position of edge points by using Laplacian operator and the output of
Laplacian is used to determine whether a point is lying on the darker side or brighter side of the edge point. From the figure 12 , it is clear that the Laplacian is negative on the brighter side and positive on the darker side. Let an image be $\mathrm{f}(\mathrm{x}, \mathrm{y})$.

$$
\begin{aligned}
& \|\nabla f\|=\left\|G_{x}\right\|+\left\|G_{x}\right\|=\sqrt{G_{x}^{2}+G_{y}^{2}} \\
& \text { Where } G_{x}=\frac{\partial f(x y)}{\partial x} \text { and } G_{y}=\frac{\partial f(x y)}{\partial y} \\
& \nabla^{2} f=\frac{\partial^{2} f}{\partial x^{2}}+\frac{\partial^{2} f}{\partial y^{2}}
\end{aligned}
$$

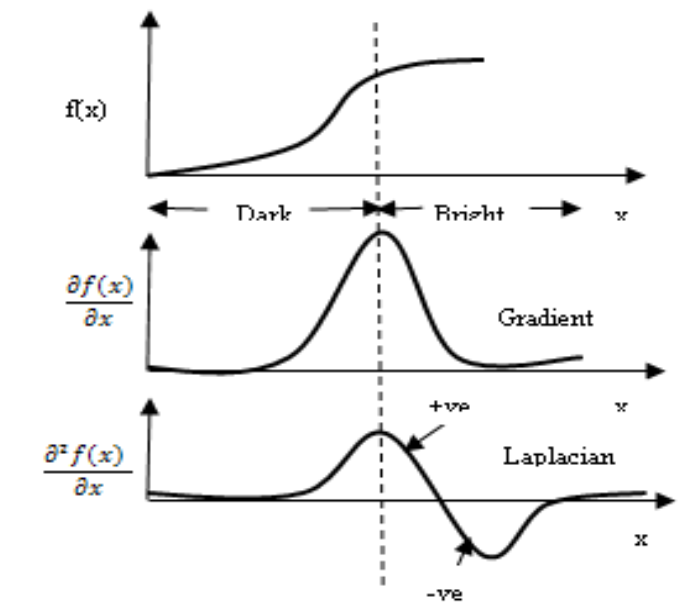

Fig. 12 Use of Laplacian and Gradient operator

If we assume that we have an image which have dark object against the bright background. In that case, for the object pixel, the Laplacian near the edge will be positive and for the background pixel, the Laplacian near the edge will be negative. Therefore, by using $\mathrm{f}(\mathrm{x}, \mathrm{y}), \| \nabla f \mid$ and $\nabla^{2} f$, we can create an image $S(x, y)$ which is given as

$$
S(x, y)=\left\{\begin{array}{lr}
0 & \text { if } \nabla f<T \\
+ & \text { if } \nabla f \geq T \text { and } \nabla^{2} f \geq 0 \\
- & \text { if } \nabla f \geq T \text { and } \nabla^{2} f<0
\end{array}\right.
$$

These three value $0,+$ and - represent three different intensity values. Then, we have to process this intermediate image $S(x, y)$ to find out the object boundary of the object region. We have scan the image either along the horizontal direction or vertical direction, we will get pattern of these three symbols $0,+$ or -. Therefore,

$(-,+) \Rightarrow$ Transition from background to object

$(+,-) \Rightarrow$ Transition from object to background

We will get sequence of symbols like that

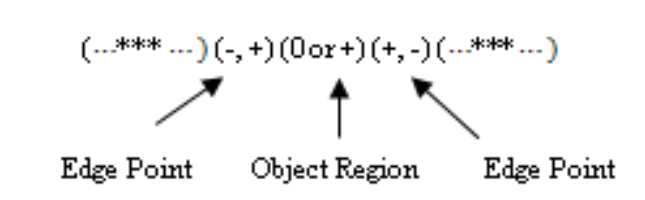

This is called local thresholding because we use gradient and Laplacian of the image and these two are local property of a particular pixel location. Figure 13 shown images which use the local thresholding. 


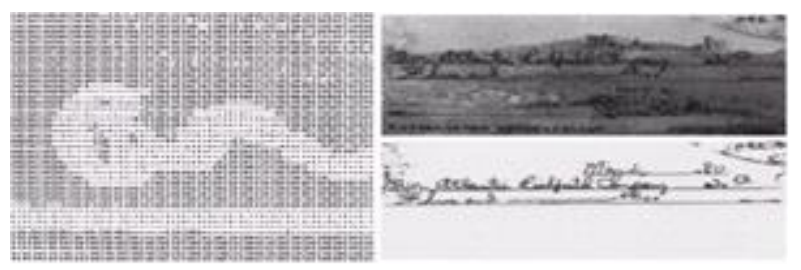

Fig. 13 Local Thresholding

In the previous sections, we have considered various images. We applied different thresholding techniques so that the image segmented well. A short summary of these thresholding techniques is shown in table I.

\section{RESULTS AND DISCUSSIONS}

\begin{tabular}{|c|c|c|c|}
\hline $\begin{array}{c}\text { Type of } \\
\text { Thresholding }\end{array}$ & $\begin{array}{l}\text { Threshold } \\
\text { value }(\mathbf{T})\end{array}$ & Advantages & Disadvantages \\
\hline $\begin{array}{l}\text { Single-Thresho } \\
\text { lding }\end{array}$ & 80 & $\begin{array}{l}\text { (1) Simple. } \\
\text { (2) Give best results in case of uniform image. } \\
\text { (3) Only one threshold value is required to segment the } \\
\text { image. } \\
\text { (4) Computational time is less. }\end{array}$ & (1) Poor results in case of non-uniform image. \\
\hline $\begin{array}{l}\text { Multiple- } \\
\text { Thresholding }\end{array}$ & {$[115,125]$} & $\begin{array}{l}\text { (1) Give best results in case of multi-model histogram. } \\
\text { (2) Computational time is less. }\end{array}$ & $\begin{array}{l}\text { (1) Give poor results when there is variation from bright to dark or } \\
\text { vice-verse in the image. }\end{array}$ \\
\hline $\begin{array}{l}\text { Adaptive } \\
\text { Thresholding }\end{array}$ & $\begin{array}{l}{[0.3255} \\
0.4627 \\
0.6667 \\
0.8314]\end{array}$ & $\begin{array}{l}\text { (1) Give best results in case of variations in gray-scale } \\
\text { image. } \\
\text { (2) By sub-dividing the image, we get separate threshold } \\
\text { to each region. }\end{array}$ & $\begin{array}{l}\text { (1) Position-dependent. } \\
\text { (2) Does tell the accuracy of the threshold. } \\
\text { (3) More computational time is required. }\end{array}$ \\
\hline $\begin{array}{l}\text { Optimal } \\
\text { Thresholding }\end{array}$ & 104.3255 & $\begin{array}{l}\text { (1) Use the statistical properties of an image. } \\
\text { (2) Gives the accurate threshold. }\end{array}$ & $\begin{array}{l}\text { (1) Does not work well in case of unequal distribution of pixels. } \\
\text { (2) Difficult to obtain because the boundary itself is not known. } \\
\text { (3) More computational time is required. }\end{array}$ \\
\hline $\begin{array}{l}\text { Local } \\
\text { Thresholding }\end{array}$ & 0.3412 & $\begin{array}{l}\text { (1) Gives good results in case of unequal distribution of } \\
\text { pixels. } \\
\text { (2) Use local properties (Laplacian and Gradient) of a } \\
\text { pixel. }\end{array}$ & $\begin{array}{l}\text { (1) Complicated } \\
\text { (2) More computational time is required. }\end{array}$ \\
\hline
\end{tabular}

\section{CONCLUSION}

In this paper, we have analyzed and evaluated the various types of thresholding techniques. Single-thresholding uses the bi-model histogram which gives the single threshold value. It is very simple approach and it works well in case of uniform image. This technique has failed when there is multi-model histogram. So, to overcome to this problem, we use multiple thresholding. Automatic thresholding automatically calculate the value of threshold. But, experimental results show that whether we calculate the value of threshold automatically or manually, it gives almost same results. If we sub-divide the image and take the separate threshold for each region and then, take the union of all these thresholds, we will get adaptive threshold. But, it also does not tell the accuracy of the threshold. So, in order to find the accurate threshold value, we use the optimal thresholding. In that case, we find the optimum threshold. Suppose, when there are very small object pixels and a large number of background pixels in an image, then, optimal thresholding does not work well because it is difficult to obtain the boundary itself. To overcome this problem, we use the local properties (Laplacian and Gradient) of the pixels to find the threshold value which is called local thresholding. In short,

all these techniques work well is some situations and does not work well in other situations. Therefore, based on the given image, we can segment the image by using these thresholding techniques.

\section{ACKNOWLEDGMENT}

The first author is grateful to the Principal of GHSS, Nilokher for their support throughout this research work. The second author is thankful to the management of Botho University for providing their support.

\section{REFERENCES}

[1] J. S. Weszka and A. Rosenfeld, "Threshold Evaluation Techniques", IEEE Transactions on Systems, Man, and Cybernetics, vol. smc-8, no. 8, Jan, 1978.

[2] N. Otsu, "A Threshold Selection Method from Gray-Level Histograms", IEEE Transactions on Systems, Man, and Cybernetics, vol. smc-9, no. 1, Jan, 1979.

[3] I. E. Abdou and W. K. Pratt, "Quantitative Design and Evaluation of Enhancement/Thresholding Edge Detectors", Proceedings of the IEEE, vol. 67 , no. 5, May, 1979.

[4] K. S. Fu, “A survey on image segmentation”, Pattern Recognition, vol 13, pp. 3-16, 1981

[5] R. M. Haralick, "Digital Step Edges from Zero Crossing of Second Directional Derivatives", IEEE Transactions on Pattern Analysis and Machine Intelligence, vol. Pami-6, no. 1, Jan. 1986.

[6] N. R. Pal and S. K. Pal, "A Review on Image Segmentation Techniques”, Pattern Recognition, vol. 26, no. 9, pp. 1277-1294, 1993.

[7] J. C. Yen, F. J. Chang and S. Chang, "A New Criterion for Automatic Multilevel Thresholding", IEEE Transactions on Image Processing, vol. 4, no. 3, Mar, 1995.

[8] A.T. Abak, U. Baris and B. Sankur, "The Performance Evaluation of Thresholding Algoithms for Optical Character Recognition”, 1997 IEEE.

[9] Francis H. Y. Chan, F. K. Lam, and Hui Zhu, "Adaptive Thresholing by Variational Method", IEEE Transaction on Image Processing, vol. 7, no. 3, Mar, 1998.

[10] C. Hampton, T. Persons, C. Wyatt and Y. Zhang, "Survey of Image Segmentation", April 23, 1998.

[11] M. Cheriet, J. N. said and C. Y. Suen, "A Recursive Thresholding Techniques for Image Segmentation”, IEEE Transaction on Image Processing, vol. 7, no. 6, Jun, 1998.

[12] O. J. Tobias and R. Seara, "Image segmentation by Histogram Thresholding using Fuzzy Sets", IEEE Transactions on Image Processing, vol. 11, no. 12, Dec, 2002.

[13] L. Djerou, H. Dehimi, N. Khelil and M. Batouche, "Self-adaptive Thresholding for Image Segmentation", Image' 09, Biskra.

[14] P. D. Satya and R. Kayalvizhi, "Development of a new Optimal Thresholding using Improved Particle Swarm Optimization Algorithm 
for Image Segmentation", International Journal of Electronics Engineering, 2(1), 2010, PP. 63-67.

[15] N. Natrajan, P. Seetal and G. S. Kumar, "Robust Image Segmentation based on Optimal Thresholding", International Journal of Engineering Studies, vol. 2, no. 2 (2010). Pp. 135-144.

[16] T. Zuva, O. O. Olugbara, S. O. Ojo and S. M. Ngwira, "Image Segmentation, Available Techniques, Developments and Open Issues", Canadian Journal on Image Processing and Computer Vision, vol. 2, no. 3, Mar, 2011

Gurjeet Kaur She has done Master of Technology in Computer Science and Engineering from the Maharishi Dayanand University, Rohtak, India in the year 2013. She is working as a computer faculty in GHSS, Nilokher since 2015. She has published more than 10 research papers in various journals and/or conferences in the field of computer science. Her area of interest is image processing.

Rajiv Kumar He has completed $\mathrm{PhD}$ in computer science from the Bharthiar University, Coimbatore, India in 2015. Currently, he is working as an Assistant Professor in the Faculty of Computing, Botho University, Gaborone, Botswana. He has published more than 20 research papers in various journals and/or conferences. His area of research interest is image processing, pattern recognition and data mining. 